\title{
Pilot-scale produced fermented lentil protects against $t$-BHP-triggered oxidative stress by activation of Nrf2 dependent on SAPK/JNK \\ phosphorilation
}

Sara Bautista-Expósito, Elena Peñas, Juana Frias, Cristina Martínez-Villaluenga*

Institute of Food Science, Technology and Nutrition (ICTAN-CSIC), Juan de la Cierva 3, 28006, Madrid.

*Corresponding author details:

Cristina Martinez Villaluenga

E-mail address: c.m.villaluenga@csic.es (C. Martínez-Villaluenga)

Mailing address: C/ Juan de la Cierva 328006 Madrid

Telephone: (+34) 912547601

Fax: (+34) 915644853 


\section{ABSTRACT}

2 Fermented lentil (FL) produced using L. plantarum and Savinase in alkaline conditions relieves

3 metabolic alterations and oxidative stress in Zucker rats with metabolic syndrome. Here, we

4 investigated the effect of up scaling the fermentation process on chemical composition and

5 biological activity of FL. Moreover, we studied the molecular mechanisms by which FL exert a

6 cytoprotective effect against oxidative stress in tert-butyl hydroperoxide ( $t$-BHP)-challenged

7 RAW264.7 macrophages. Up-scale production reduced overall biological effectiveness of FL

8 with the exception of inhibition of intracellular ROS generation. FL prevented $t$-BHP-induced

9 cytotoxicity and intracellular accumulation of reactive oxygen species through activation of

10 catalase expression via SAPK/JNK phosphorylation and Nrf2 nuclear translocation. Different

11 oligopeptides, phenolic acids and flavonols were identified as contributors of the observed

12 effects. To the best of our knowledge, we reported for the first time that FL attenuates oxidative

13 stress cellular damage via activation of SAPK/JNK phosphorylation, Nrf2 nuclear tranlocation

14 and antioxidant enzymes expression.

15 Keywords: Nfr2, lentil, digestion, peptides, phenolic compounds, oxidative stress 
The exponential increase of people affected by metabolic syndrome (MetS) has led the

18 scientific community to explore new lifestyle interventions that effectively revert all components

19 of this disease including central obesity, dyslipemia, elevated plasma glucose, blood pressure

20 and insulin resistance (Grundy, 2016). Patients with MetS are more prone to develop type 2

21 diabetes mellitus, cardiovascular diseases and non-alcoholic fat liver disease (NAFLD).

22 Impaired antioxidant status and consequent development of oxidative stress are also intimately

23 related to MetS and its associated comorbidities (Zaki et al., 2018). Reactive oxygen species

24 (ROS) mediate various signaling networks exerting cytotoxic effects, such as peroxidation of

25 lipids and phospholipids and oxidative damage to proteins and DNA, leading to tissue 26 destruction (Zaki et al., 2018).

Legume interventions become a subject of increasing interest since they have shown

28 beneficial properties related to the management of MetS (Martínez, López-Jurado, Wanden-

29 Berghe, Sanz-Valero, Porres, \& Kapravelou, 2016). Legume intake has positive effects including

30 glycemia and lipemia decrease and improvement of insulin sensitivity, weight management and

31 satiety perception (Clark \& Duncan, 2017; Mollard, Luhovyy, Panahi, Nunez, Hanley, \&

32 Anderson, 2012). Beside fiber and oligosaccharides, other bioactive compounds such as phenolic

33 compounds and peptides have emerged as contributors to the positive effects of legume intake

34 on MetS (Padhi, Liu, Hernandez, Tsao, \& Ramdath, 2017; Padhi \& Ramdath, 2017; Reverri,

35 Randolph, Kappagoda, Park, Edirisinghe, \& Burton-Freeman, 2017). Health promoting

36 properties of phenolic compounds and bioactive peptides of legumes come from their

37 physiological effects such as modulation of blood pressure as well as glucose and lipid

38 homeostasis given that they inhibit the activity of angiotensin converting enzyme I, intestinal $\alpha$ -

39 glucosidase, pancreatic $\alpha$-amylase and lipase (Iwaniak, Darewicz, \& Minkiewicz, 2018; Zhang 
40 et al., 2015). They may also act as antioxidants providing protection against oxidative stress

41 (García-Mora et al., 2017; Padhi, Liu, Hernandez, Tsao, \& Ramdath, 2017).

Different technological strategies including germination and fermentation have been

43 employed to exploit legumes as functional foods for the management of MetS (Bautista-

44 Expósito, Peñas, Silván, Frias, \& Martínez-Villaluenga, 2018a; Kapravelou et al., 2017). We

45 have recently developed a laboratory scale fermentation in alkaline conditions with

46 Lactobacillus plantarum CECT 748 and the enzymatic preparation Savinase 16 L to increase the

47 bioavailability of bioactive peptides and phenolics while enhancing the antioxidant,

48 hypoglucemic, hypolipidemic, anti-inflammatory and anti-hypertensive activities of lentil (Lens

49 culinaris Medik. var. Castellana) (Bautista-Expósito et al. 2018a). We demonstrated the

50 individual effect of Savinase, mainly producing bioactive peptides, and L. plantarum responsible

51 for the increased concentrations of $p$-hydroxybenzoic acid, vanillic acid and kaempferol

52 glucosides in the end-product (Bautista-Expósito, Peñas, Dueñas, Silván, Frias, \& Martínez-

53 Villaluenga, 2018b). Due to this lentil fermentation procedure is intended to be used for

54 obtaining a new functional ingredient against MetS, we have studied herein the effect of up

55 scaling the process on bioactive peptides, phenolics and bioactivity of the end-product.

The beneficial effects of fermented lentil (FL) developed by our research group were

57 recently validated in the obese Zucker rat experimental model of MetS and NAFLD (Martinez

58 et al., 2018). Administration of FL effectively reduced steatosis through the expression of genes

59 involved in hepatic $\beta$-oxidation. Moreover, FL improved glucose and lipid metabolism and

60 reduced lipid peroxidation in the liver of Zucker rats through increased gene expression of

61 nuclear factor-erythroid-2-related transcription factor-2 (Nrf2). This transcription factor controls

62 the expression of genes whose protein products are involved in the detoxication and elimination

63 of reactive oxidants and electrophilic agents (Nguyen, Nioi, \& Pickett, 2009). Since regulatory

64 mechanisms of $\mathrm{Nfr} 2$ activation by FL have not been elucidated yet, in this study, we 
65 hypothesized that bioactive peptides and phenolic compounds present in FL activate redox

66 signaling networks, induce Nrf2 translocation to the nucleus and subsequently the expression of

67 antioxidant enzymes that would contribute to reduction of intracellular ROS levels and cell

68 survival. To test this hypothesis, we performed in vitro experiments in which we simulated the

69 gastrointestinal digestion of FL in physiological conditions and subsequently studied the

70 antioxidant effects and mechanism of action of FL digest (FLD) using tert-butyl hydroperoxide-

71 challenged RAW 264.7 cells as a model of oxidative stress.

72 The aims of this study were: i) to scale-up fermentation process to determine the

73 feasibility of producing enough quantities of FL to be included as an ingredient in foods; ii) to

74 evaluate the protective role ofFLD on tert-butyl hydroperoxide ( $t$-BHP) induced oxidative stress

75 in RAW 264.7 macrophages; iii) to elucidate the molecular mechanisms involved in the

76 protective effect of FLD against oxidative stress; iv) to characterize the peptide and phenolic

77 profiles of FLD.

\section{2. Material and Methods}

79 2.1.Materials

80 Lentil seeds (Lens culinaris Medik. var. Castellana) were obtained from Semillas Iglesias S.A.

81 (Salamanca, Spain). A commercial food grade protease Savinase ${ }^{\circledR} 16 \mathrm{~L}(16 \mathrm{KNPU} / \mathrm{g})$, an

82 enzymatic preparation of alkaline-serine protease from Bacillus spp. was provided by

83 Novozymes (Bagsvaerd, Denmark). L. plantarum CECT 748 was purchased from the Spanish

84 Type Culture Collection (Valencia, Spain).

\section{2.2. Chemicals}

86 Angiotensin I converting enzyme (ACE, EC 3.4.15.1), rat intestine $\alpha$-glucosidase (EC 3.2.1.20),

87 porcine pancreatic lipase type II (EC 3.1.1.3), pepsin from porcine gastric mucosa (EC 3.4.23.1), 88 pancreatin from porcine pancreas (EC 232-468-9), 2,7-dichlorofluorescein diacetate (DCFDA) 
89 and $t$-BHP were purchased from Sigma-Aldrich (Madrid, Spain). Murine macrophage cell line

90 RAW 264.7 was obtained from the American Type Culture Collection (Rockville, MD, USA).

91 High-glucose Dulbecco's Modified Eagle's Medium (DMEM) and penicillin/streptomycin

$92(10,000 \mathrm{U} / \mathrm{mL})$ were purchased from Lonza Group (Madrid, Spain). Fetal bovine serum (FBS)

93 was obtained from Hyclone (GE Healthcare, Logan, UT, USA). Primary antibodies for Cu-Zn

94 superoxide dismutase (SOD-1), catalase and $\beta$-actin were obtained from Santa Cruz

95 Biotechnology (Santa Cruz, CA, USA), rabbit transcriptional activator Nrf2, Lamin B1, stress-

96 activated protein kinases (SAPK)/Jun amino-terminal kinases (JNK) and phosphorylated

97 SAPK/JNK in Thr183/Tyr185 were from Cell Signaling Technologies (Danvers, MA, USA).

98 Secondary antibodies [horseradish peroxidase (HRP)-conjugate recombinant mouse

99 immunoglobulin $\mathrm{G}$ kappa (m-IgGK) light chain binding protein and HRP-conjugate goat anti-

100 rabbit IgG (heavy and light chain)] were obtained from Santa Cruz Biotechnology and Cell

101 Signaling, respectively.

\section{2.3. Scale-up of lentil flour fermentation}

103 To scale-up production, in this study FL was produced in a $30 \mathrm{~L}$ final volume using a $50 \mathrm{~L}$

104 BIOSTAT $^{\circledR}$ D bioreactor (Sartorious, Barcelona Spain). Lentil flour was suspended in 105 autoclaved water (142.7 g flour/L), inoculated with Lactobacillus plantarum CECT $748\left(2 \times 10^{8}\right.$

$106 \mathrm{CFU} / \mathrm{L})$ and simultaneously treated with Savinase $16 \mathrm{~L}(361 \mathrm{mg} / \mathrm{L}$ lentil flour suspension).

107 Treatment was performed at $37{ }^{\circ} \mathrm{C}$ and $\mathrm{pH} 8.0$ (adjusted using $20 \% \mathrm{KOH}$ ), continuous stirring

108 speed of $300 \mathrm{rpm}$, without aeration for $15 \mathrm{~h}$. Flour suspension was centrifuged and the obtained

109 soluble fraction was frozen at $-80{ }^{\circ} \mathrm{C}$ and freeze-dried using FreeZone $12 \mathrm{~L}$ freeze-dryer

110 (Labconco, Kansas, MO, USA).

111 2.4. Gastrointestinal digestion 
112 FL was digested following an in vitro harmonized protocol that reached an international

113 consensual agreement (Minekus et al., 2014). Briefly, freeze-dried lentil samples were dissolved

114 in water $(700 \mathrm{mg} / 5 \mathrm{~mL})$ and then, diluted with simulated gastric fluid containing pepsin (2000

$115 \mathrm{U} / \mathrm{mL}$ in the final mixture, ratio of $50: 50, \mathrm{v} / \mathrm{v})$. The mixture was incubated at $37{ }^{\circ} \mathrm{C}$ in an orbital

116 shaker at $150 \mathrm{rpm}$ for $120 \mathrm{~min}$. The enzymatic reaction was stopped by adjusting the $\mathrm{pH}$ at 7.0

117 with $1 \mathrm{M} \mathrm{NaOH}$. Gastric phase was mixed with simulated intestinal fluid containing pancreatin

118 (100 U trypsin activity $/ \mathrm{mL}$ of final mixture, ratio 50:50, v/v) and porcine bile extract (10 $\mathrm{mM}$ in

119 the final mixture). Intestinal digestion phase was carried out at $37^{\circ} \mathrm{C}$ and $150 \mathrm{rpm}$ for $120 \mathrm{~min}$.

120 The enzymatic reaction was stopped at $85^{\circ} \mathrm{C}$ for $10 \mathrm{~min}$. Digestion was performed in duplicate.

121 FLD was freeze dried and kept at $-20{ }^{\circ} \mathrm{C}$ in vacuum sealed plastic bags until further analysis.

122 2.5. Determination of total peptides and phenolic compounds

123 Peptide concentration was measured by Pierce Quantitative Colorimetric Peptide Assay kit

124 (Fisher Scientific, Madrid, Spain) in permeates obtained by ultrafiltration through cellulose

125 membranes of $10 \mathrm{kDa}$ pore size (Millipore, Billerica, MA). Results were expressed as mg of

126 peptides/g of soluble fraction. Total phenolic compounds were determined using the Folin-

127 Ciocalteau method as previously described (Garcia-Mora et al., 2015). The absorbance was read

128 at $690 \mathrm{~nm}$ using a Synergy HT multi-well plate reader (BioTek, Winooski, VT, USA) and the

129 results were expressed as mg gallic acid equivalents (GAE)/g of soluble fraction.

\section{2.6. Peptides identification}

131 A peptidomic analysis was performed to identify the peptides present in FLD. Twenty mg of 132 freeze-dried sample were suspended in $1 \mathrm{~mL}$ of $0.1 \%$ trifluoroacetic acid (TFA) using and 133 ultrasound bath. An aliquot $(5 \mu \mathrm{L})$ was 5 -fold diluted in $0.1 \%$ TFA. From this sample solution $1342.5 \mu \mathrm{L}$ were purified using a reverse phase C18 ZipTip (Millipore Corporation, MA, USA). 135 Eluate was resuspended in $0.1 \%$ formic acid (FA) $/ 2 \%$ acetonitrile (ACN) and injected ( $4 \mu \mathrm{L})$ in 
136 an Easy liquid chromatography 1000 nano system (Thermo Fisher Scientific, Madrid, Spain)

137 coupled to an ion source with nanoelectrospray LTQ-Orbitrap Velos (Thermo Fisher Scientific,

138 Madrid, Spain). For each analysis, the sample was loaded into a precolumn Acclaim PepMap

139100 (Thermo Fisher Scientific, Madrid, Spain) and eluted in an AcclaimPepMap 100 C18 (250

$140 \mathrm{~mm}$ long, $75 \mu \mathrm{m}$ inner diameter and $3 \mu \mathrm{m}$ particle size; Thermo Fisher Scientific, Madrid, Spain).

141 The mobile phase flow rate was $250 \mathrm{~nL} / \mathrm{min}$ using $0.1 \% \mathrm{FA}$ in water (solvent $\mathrm{A}$ ) and $0.1 \% \mathrm{FA}$

142 in ACN (solvent B). The gradient profile was set as follows: 0\%-35\% solvent B in 90 min, 35\%-

$14345 \%$ solvent B in 10 min, $45 \%-95 \%$ solvent B in 5 min; $95 \%-100 \%$ solvent B in 1 min, $100 \%$

144 solvent B in 4 min.

145 Mass spectrometry (MS) analysis was performed using a LTQ-Orbitrap Velos working in the

146 positive mode. For ionization, $2000 \mathrm{~V}$ of liquid junction voltage and $270{ }^{\circ} \mathrm{C}$ capillary

147 temperature was used. The full scan method employed a $\mathrm{m} / \mathrm{z}$ 400-2000 mass selection, an

148 Orbitrap resolution of 60,000 (at $\mathrm{m} / \mathrm{z} 200$ ), a target automatic gain control (AGC) value of $3 \mathrm{e}^{6}$,

149 and maximum injection times of $100 \mathrm{~ms}$. After the survey scan, the 15 most intense precursor

150 ions were selected for ion fragmentation. Fragmentation was performed by collision-induced

151 dissociation in the ionic tramp using a normalized collision energy of $35 \%$. MS/MS scans were

152 acquired using a starting mass of $\mathrm{m} / \mathrm{z} 100$, AGC target of $2 \mathrm{e}^{5}$, resolution of $17,500($ at $\mathrm{m} / \mathrm{z} 200)$,

153 intensity threshold of $8 \mathrm{e}^{3}$, isolation window of $2 \mathrm{~m} / \mathrm{z}$ units and maximum injection time of 100

154 ms. Charge state screening was enabled to reject unassigned, singly charged, and equal or more

155 than seven protonated ions. A dynamic exclusion time of $45 \mathrm{~s}$ was used to discriminate against

156 previously selected ions.

157 MS data were analyzed with Proteome Discoverer (version 1.4.1.14) (Thermo Fisher Scientific,

158 Madrid, Spain) using standardized workflows. Mass spectra raw files were searched against Lens

159 culinaris database files (NCBI, 427 sequences protein entries) using SEQUEST search engine.

160 Precursor and fragment mass tolerance were set to $10 \mathrm{ppm}$ and $0.5 \mathrm{Da}$, respectively. In the search 
161 parameters carbamidomethylation of cysteines and methionine oxidation were established as

162 fixed modifications. Identified peptides were filtered using Percolator algorithm with a q-value 163 threshold of 0.01.

\subsection{Quantification and identification of phenolic compounds}

Freeze-dried FLD soluble fraction (50 mg) was dissolved in $1 \mathrm{~mL}$ methanol:water (50:50,

$166 \mathrm{v} / \mathrm{v})$. Sample solution was vortexed during $10 \mathrm{~min}$ and subsequently, centrifuged at 10,000 x $g$

167 and $5{ }^{\circ} \mathrm{C}$ for $10 \mathrm{~min}$. The supernatant was filtered through $0.45 \mu \mathrm{m}$ polytetrafluoroethylene 168 membrane (Agilent, Santa Clara, CA, USA). Chromatographic analyses were performed in an 169 Agilent high performance liquid chromatography (HPLC) 1100 series equipped with a a G1312A 170 binary pump, a G1313 A autosampler, G1315B photodiode array detector, controlled by the

171 Agilent software v. A.08.03 and G1322A degasser. Briefly, sample $(20 \mu \mathrm{L})$ elution was carried 172 out at a flow rate of $1 \mathrm{~mL} / \mathrm{min}$, on a Kinetex column $(5 \mu \mathrm{m}, \mathrm{C} 18,250 \times 4.6 \mathrm{~mm}$; Phenomenex, 173 Macclesfield, UK), with 1\% FA in water (solvent A) and ACN (solvent B), starting with 5\% B 174 and installing a gradient to obtain $60 \% \mathrm{~B}$ at $37 \mathrm{~min}, 98 \% \mathrm{~B}$ at $40 \mathrm{~min}$ and $98 \% \mathrm{~B}$ at $42 \mathrm{~min}$.

175 HPLC was coupled to an ion-trap mass spectrometer equipped with an electrospray ionization 176 system (ESI). The nebulizer pressure and flow rate of nitrogen were $65.0 \mathrm{psi}$ and $11 \mathrm{~L} / \mathrm{min}$, 177 respectively. The heated capillary and voltage were maintained at $350{ }^{\circ} \mathrm{C}$ and $3.5 \mathrm{kV}$, 178 respectively. Mass scan and MS/MS daughter spectra were measured from m/z 100 to 1200 .

179 Collision-induced fragmentation experiments were performed in the ion trap using helium as the 180 collision gas, and the collision energy was set at 50\%. MS data were acquired in the negative 181 ionization mode. The phenolics compounds in FLD were identified according to their UV 182 spectrum, molecular mass, daughter ions, and fragmentation pattern. For quantitative analysis, 183 calibration curves were prepared by injection of known concentrations of different standard 184 compounds obtained from Extrasynthese (Genay Cedex, France). Samples were analysed in 185 duplicate. Data were expressed as $\mu \mathrm{g} / \mathrm{g}$ of soluble fraction. 
Angiotensin I converting enzyme (ACE) inhibitory activity was measured following a 188 previously described method (Garcia-Mora, et al., 2015). Fluorescence was read every minute 189 for $30 \mathrm{~min}$ at emission and excitation wavelengths of 335 and $405 \mathrm{~nm}$, respectively, in a 190 microplate reader Synergy HT (BioTek, Winooski, VT, USA). The results were expressed as 191 percent inhibition relative to the negative control having $100 \%$ enzymatic activity. All samples 192 were analyzed in duplicate. $\mathrm{IC}_{50}$ values (concentration of sample in $\mathrm{mg} / \mathrm{mL}$ that inhibits $50 \%$ of 193 the ACE activity) were calculated plotting the non-linear regression sigmoidal dose-response 194 curves in GraphPad Prism 4.00 (GraphPad Software Inc., San Diego, CA, USA).

\section{9. $\alpha$-Glucosidase inhibitory activity}

$\alpha$-glucosidase sucrase and maltase inhibitory activities of FL $(1.7 \mathrm{mg} / \mathrm{mL}$ final

197 concentration) was determined following a previously described method (Bautista-Expósito, et

198 al., 2018a). Glucose concentration in the reaction mixture was determined using the Amplex ${ }^{\circledR}$ 199 Red glucose/glucose oxidase assay kit (Invitrogen, Carlsbad, CA, USA). Absorbance was 200 measured using a Synergy HT plate reader (BioTek, Winooski, VT, USA) at $560 \mathrm{~nm}$. Glucose 201 concentration was calculated using a linear standard curve $(0-200 \mu \mathrm{M})$ from a freshly prepared $202400 \mathrm{mM}$ stock solution. All samples were analyzed in duplicate. Percent inhibition of sucrase 203 and maltase activity of intestinal $\alpha$-glucosidase in the presence of lentil samples was calculated 204 relative to the negative control having $100 \%$ enzyme activity.

\subsection{Lipase inhibitory activity}

The ability of FL (final concentration of $2 \mathrm{mg} / \mathrm{mL}$ ) to inhibit porcine pancreatic lipase

207 was evaluated using a previously reported method (Bautista-Expósito et al. 2018a). Lipase 208 activity was determined by measuring the hydrolysis of $p$-nitrophenyl butyrate to $p$-nitrophenol 209 at $405 \mathrm{~nm}$ using a microplate reader Synergy HT (BioTek, Winooski, VT, USA). All samples 
210 were analyzed in duplicate. Percent inhibition of lipase activity in the presence of lentil samples

211 was calculated relative to the negative control having $100 \%$ enzyme activity.

\section{2.11. Oxygen radical absorbance capacity}

213 Radical scavenging activity was evaluated by fluorescence following the oxygen radical

214 absorbance capacity (ORAC) assay described previously (Bautista-Expósito et al., 2018a).

215 Fluorescence was measured in a Synergy HT microplate reader (BioTek, Winooski, VT, USA)

216 at emission and excitation wavelengths of $520 \mathrm{~nm}$ and $485 \mathrm{~nm}$, respectively. A Trolox standard

217 curve with a linear concentration range $(0-160 \mu \mathrm{M})$ was prepared from a freshly made $1 \mathrm{mM}$

218 stock solution. All samples were analyzed in duplicate. Results were expressed as mM of Trolox

219 Equivalents (TE)/g of soluble fraction.

In a previous in vivo study, it was demonstrated that fermented lentil intervention was

222 able to reduce lipid peroxidation in the liver of obese Zucker rats with non-alcoholic fatty liver

223 disease (NAFLD) by increased expression of Nfr2 (Martínez et al. 2018). To get a deeper insight

224 on the antioxidant mechanism of action of fermented lentil described in the obese Zucker rat we

225 decided to use a murine cell model of oxidative stress. We selected a murine macrophage cell

226 line, as pathophysiology of NAFLD is characterized by infiltration of macrophages to the hepatic

227 tissue increasing oxidative stress and inflammation (Dongiovanni, Lanti, Riso, \& Valenti, 2016).

228 To test the cytoprotective effect of FLD in stressful conditions, a model of oxidative 229 stress induced by a potent pro-oxidant was established based on previous studies (Lv, Liu, Zhou,

230 Tan, Deng, \& Ci, 2017). RAW 264.7 murine macrophages were cultured using DMEM

231 supplemented with $10 \%$ FBS, 1\% penicillin/streptomycin. Cells were maintained at a 232 subconfluent density in $75 \mathrm{~cm}^{2}$ tissue culture flasks and incubated in a humidified $5 \% \mathrm{CO}_{2} / 95 \%$ 
233 air incubator at $37^{\circ} \mathrm{C}$. The culture medium was changed every 2 days. In all experiments, cells

234 were allowed to acclimate for $24 \mathrm{~h}$ before treatments.

\section{2.13. Cell viability}

RAW 264.7 cells were grown in 96 -well plates $\left(5 \times 10^{4}\right.$ cells/well $)$ for $24 \mathrm{~h}$ and stimulated

237 with $t$-BHP $(0.05,0.1,1.0$ and $2.5 \mathrm{mM})$ for an additional $3 \mathrm{~h}$. Additional experiments consisted 238 of cell treatment with FLD $(50,75$ and $100 \mu \mathrm{g} / \mathrm{mL})$ for $18 \mathrm{~h}$ or FLD $(50,75$ and $100 \mu \mathrm{g} / \mathrm{mL})$ for

$23918 \mathrm{~h}$ and with $2.5 \mathrm{mM} t$-BHP for an additional $3 \mathrm{~h}$. Each treatment was performed in triplicate 240 to confirm reproducibility. Control cells incubated in serum-free medium served as negative 241 control. Cell viability was determined using the Cell Titer 96® AQueous One Solution 242 Proliferation Assay kit from Promega (Madison, WI, USA). Medium was removed, cells were 243 washed with phosphate buffer saline (PBS) and $100 \mu \mathrm{L}$ of serum-free medium were added. 244 Thereafter, $20 \mu \mathrm{L}$ of Cell Titer $96{ }^{\circledR}$ were added to each well for the quantification of the living 245 metabolically active cells. After 45 min of incubation, absorbance was read at $515 \mathrm{~nm}$ employing 246 a PowerWave ${ }^{\mathrm{TM}}$ XS microplate reader (BioTek, Winooski, VT, USA). The viability was

247 calculated considering negative control (non-treated cells) as 100\% viable.

\subsection{Detection of intracellular ROS}

Intracellular ROS were quantified by the dichlorofluorescein (DCF) assay. Macrophages

$250\left(2 \times 10^{5}\right.$ cells/well $)$ were grown in clear bottom black 24 well-plates for $24 \mathrm{~h}$. Cells were washed 251 with PBS and incubated with $20 \mu \mathrm{M}$ DCFDA for $30 \mathrm{~min}$. After that, cells were washed with PBS 252 and treated with $t$-BHP $(0.05,0.1,1.0$ and $2.5 \mathrm{mM})$ for an additional $3 \mathrm{~h}$. Additional experiments 253 consisted of cell treatment with FLD $(50,75$ and $100 \mu \mathrm{g} / \mathrm{mL})$ for $18 \mathrm{~h}$ or FLD $(50,75$ and 100 $254 \mu \mathrm{g} / \mathrm{mL}$ ) for $18 \mathrm{~h}$ and $2.5 \mathrm{mM} t$-BHP for an additional $3 \mathrm{~h}$. Each treatment was performed in 255 triplicate to confirm reproducibility. Control cells incubated in serum-free medium served as 256 negative control. ROS production was monitored for $3 \mathrm{~h}$ by fluorescence at an excitation and 
257 emission wavelengths of $485 \mathrm{~nm}$ and $530 \mathrm{~nm}$, respectively, using a Synergy HT plate reader

258 (BioTek, Winooski, VT, USA).

259 2.15. Whole cell lysates preparation

260 Upon completion of treatments, cells were washed with $500 \mu \mathrm{L}$ of ice cold PBS to remove

261 cellular debris. Cells were lysed with $100 \mu \mathrm{L}$ RIPA lysis buffer containing protease and

262 phosphatase inhibitors cocktail (Santa Cruz Biotechnology, Santa Cruz, CA, USA) and

263 incubated for $5 \mathrm{~min}$ at $4{ }^{\circ} \mathrm{C}$. The cells were dislodged using cell scrapers and vortexed for $5 \mathrm{~min}$

264 at $4{ }^{\circ} \mathrm{C}$ followed by centrifugation at $14,000 \mathrm{x} g$ for $10 \mathrm{~min}$ at $4{ }^{\circ} \mathrm{C}$. The pellet was discarded and

265 the cell supernatant was transferred into pre-chilled tubes. The protein concentration of the whole

266 cell lysates was measured using the Detergent Compatible Protein assay kit (Bio-Rad

267 Laboratories, Hercules, CA, USA). Whole cell lysates were stored at $-80^{\circ} \mathrm{C}$ until use.

268 2.16. Preparation of cytosolic and nuclear fractions

269 For Nrf2 and Lamin B1 Western blot analysis, nuclear proteins were extracted after $18 \mathrm{~h}$

270 of FLD treatment $(50,75$ and $100 \mu \mathrm{g} / \mathrm{mL})$. For Western blot analysis of phosphorylated (P)-

$271 \mathrm{SAPK} / \mathrm{JNK}$ and total (T)-SAPK/JNK, cytoplasmic proteins were extracted after $6 \mathrm{~h}$ of FLD

272 treatment $(50,75$ and $100 \mu \mathrm{g} / \mathrm{mL})$. Upon completion of treatments, cells were subsequently

273 washed with $500 \mu \mathrm{L}$ of ice cold PBS to remove cellular debris. To separate cytoplasmic and

274 nuclear fractions, NE-PER nuclear extraction kit (Thermo Fisher Scientific, Madrid, Spain) was

275 used following manufacturer's instructions. All steps were carried out on ice or at $4{ }^{\circ} \mathrm{C}$. The

276 protein concentration of nuclear and cytosolic fractions was measured using the Detergent

277 Compatible Protein assay kit (Bio-Rad Laboratories, Hercules, CA, USA). Nuclear or

278 cytoplasmic fractions were stored at $-80^{\circ} \mathrm{C}$ until use. 
280 Expressions of SOD-1, catalase and $\beta$-actin were measured by western blot in the whole cell 281 lysates. Nrf2 and Lamin B1 were measured in the nuclear fraction while P-SAPK/JNK and T$282 \mathrm{SAPK} / \mathrm{JNK}$ were measured in cytosolic fraction. Sodium-dodecyl sulfate polyacrylamide gel 283 electrophoresis (SDS-PAGE) was run under denaturing conditions with $20 \mu \mathrm{g}$ of protein loaded 284 in $4-20 \%$ on NuPAGE ${ }^{\circledR}$ Novex $4-12 \%$ Bis-Tris Gels (Invitrogen, Madrid, Spain). Gels were 285 placed in XCell-sure lock Mini-Cell and run under reducing conditions at $200 \mathrm{~V}$. NuPAGE ${ }^{\circledR}$ 286 MES-SDS and NuPAGE ${ }^{\circledR}$ LDS (Invitrogen, Madrid, Spain) were used as running and sample 287 buffers, respectively. After protein separation by SDS-PAGE and transfer, polyvinylidene 288 fluoride (PVDF) membranes (GE Healthcare, Logan, UT, USA) were blocked with 5\% non-fat 289 dry milk for $1 \mathrm{~h}$ at room temperature and washed with Tris buffer saline Tween 20 (TBST), three

290 times for 10 min each. PVDF membranes were incubated in primary antibodies against SOD-1, 291 catalase and $\beta$-actin, Nrf2 and Lamin B1, P-SAPK/JNK and T-SAPK/JNK overnight at $4{ }^{\circ} \mathrm{C}$. 292 After washing, secondary antibodies were added and incubated at room temperature for $2 \mathrm{~h}$. 293 After incubation and repeated washing, the PVDF membranes were prepared for detection using 294 a 1:1 mixture of chemiluminescent reagent A (luminol solution) and B (peroxide solution) (GE 295 Healthcare, Logan, UT, USA). The membrane pictures were taken on a ChemDoc XRS+ 296 Imaging System (Bio-Rad Laboratories, Hercules, CA, USA). The relative amount of each target 297 protein was normalized to $\beta$-actin. For the detection of Nrf2, equal amount of protein $(15 \mu \mathrm{g})$ 298 from nuclear extracts was normalized to Lamin B1. All Western blot procedures were 299 determined in at least three independent trials.

\subsection{Statistical analysis}

Experimental data represent the mean and standard deviation of three replicates analyzed in

302 duplicate. Statistical significance of the data was tested by one-way analysis of variance, 303 followed by the Duncan test to compare the means that showed significant variation $(\mathrm{p}<0.05)$; 
304 all of the statistical analyses were performed using Statgraphics Plus software, version 5.1

305 (Statistical Graphics Corporation, Rockville, MD, USA).

\section{3. Results}

307 3.1. Effect of scale up on peptide and phenolic yields and bioactivity profile of FL

In an earlier study, FL was produced at laboratory scale using L. plantarum CECT 748

309 along with Savinase $16 \mathrm{~L}$ at $\mathrm{pH} 8.5$ for $15 \mathrm{~h}$ to enhance the multifunctional potential of lentil in

310 relation to the management of hypertension, hyperglycemia, dislipemia and oxidative stress

311 (Bautista-Expósito et al., 2018a). In the present study, we scale up FL production from 1.5 L to

$31230 \mathrm{~L}$ final volume with some minor modifications. As sodium may have a negative impact in

313 blood pressure (Bazzano, Green, Harrison, \& Reynolds, 2013), NaOH was substituted by $\mathrm{KOH}$

314 to adjust $\mathrm{pH}$ value during fermentation. Pilot scale fermentation was performed at $\mathrm{pH} 8.0$ instead

315 of $\mathrm{pH} 8.5$, therefore, lower volume of alkali solution $(20 \% \mathrm{KOH})$ were required to adjust the $\mathrm{pH}$

316 value.

317 Peptide and phenolic yields as well as bioactivity of FL produced at lab-scale and pilot-

318 scale were compared as it is shown in Table 1. FL produced at pilot plant scale was comprised

319 of $480.4 \mathrm{mg}$ protein/g, $181.3 \mathrm{mg}$ peptides/g and $49.2 \mathrm{mg} \mathrm{GAE} / \mathrm{g}$. As compared to lab-scale, FL

320 production at $30 \mathrm{~L}$ scale significantly reduced protein content, peptide and phenolic yields

321 ( $p<0.05)$. Similarly, FL bioactivity was significantly lower when the fermentation process was

322 scaled up to $30 \mathrm{~L}$. Lower inhibition of ACE, sucrase activity of intestinal $\alpha$-glucosidase and 323 pancreatic lipase as well as ORAC values were observed for FL produced at pilot scale. On the

324 contrary, scale up of fermentation process did not affect maltase activity of FL and noticeably 325 increased the effectiveness of FL to protect RAW 264.7 macrophages against oxidative stress 326 ( $>0.05$; Table 1). Based on these results, to get a deeper insight in the protective effects of FL 327 against oxidative stress we further performed an in vitro gastrointestinal digestion of FL to mimic 
328 physiological processes that upon intake have an impact on chemical composition and

329 bioactivity. This experimental approach allowed us to compare our results with those previously

330 observed in Zucker rats with MetS (Martinez et al., 2018). Thus, subsequent experiments were

331 performed to investigate the protective effect and mechanisms of action of FLD in $t$-BHP-

332 induced RAW 264.7 macrophages.

333 3.2. FLD protect RAW264.7 cells against $t$-BHP-induced cytotoxicity

334 To test the cytoprotective effect of FLD, a model of oxidative stress induced by a potent pro335 oxidant such as $t$-BHP was used based on previous studies (Lv, Liu, Zhou, Tan, Deng, \& Ci, 336 2017). $t$-BHP can induce cell damage, inhibiting cell viability and inducing cell apoptosis.

337 Different $t$-BHP concentrations $(0.05,0.1,1$ and $2.5 \mathrm{mM})$ were tested to assure that toxicity by 338 oxidative stress on RAW 264.7 macrophages was produced. Results show that $0.1,1.0$ and 2.5 $339 \mathrm{mM}$ of $t$-BHP resulted in a remarkable decrease of cell viability $(78 \%, 39 \%$ and $11 \%$ 340 respectively, Figure 1A). Subsequently, macrophages were exposed to increasing concentrations 341 of FLD $(10,50,75$ and $100 \mu \mathrm{g} / \mathrm{mL})$ during $18 \mathrm{~h}$ and cell viability was measured. Cell viability 342 of FLD-treated cells was not significantly different compared to control cells $(\mathrm{p}>0.05$, Figure 343 1B), therefore, none of the tested concentrations of FLD induced cytotoxicity. To determine 344 whether treatment with FLD has a cytoprotective effect against stressful oxidant conditions, cells 345 were treated with FLD $(10,50,75$ and $100 \mu \mathrm{g} / \mathrm{mL})$ for $18 \mathrm{~h}$ and subsequently exposed to 2.5 $346 \mathrm{mM} \mathrm{t}$-BHP for $3 \mathrm{~h}$. Results showed that FLD at 75 and $100 \mu \mathrm{g} / \mathrm{mL}$ significantly inhibited $t$-BHP 347 induced cell death (42\% and 61\%, respectively, Figure 1C).

$349 t$-BHP exposure to RAW 264.7 cells increased in a dose-dependent manner the production of

350 ROS (Figure 1D). Levels of oxidant radicals in cells treated with increasing concentrations of 351 FLD did not show significant differences compared to control untreated cells $(\mathrm{p}>0.05$, Figure 
352 1E). These results indicate that FLD alone did not induce oxidative stress to RAW 264.7 353 macrophages. Pretreatment of RAW 267.4 cells with FLD $(10,50,75$ and $100 \mu \mathrm{g} / \mathrm{mL})$ for $18 \mathrm{~h}$

354 followed by $2.5 \mathrm{mM}$ exposure of $t$-BHP for $3 \mathrm{~h}$ dose-dependently reduced the generation of

355 oxidant radicals $(3,19,32$ and $67 \%$ inhibition, respectively, Figure 1F).

\subsection{FLD induces catalase protein expression}

357 To elucidate the possible molecular pathways of the anti-oxidant role of FLD, the protein 358 expression of antioxidant enzymes (catalase and SOD-1) by Western blot was evaluated (Figure

359 2A). Cell exposure to $0.1 \mathrm{mM} t$-BHP increased catalase and SOD-1 levels compared to control 360 untreated cells ( $p<0.05$, Figure 2B and 2C). The choice of $t$-BHP dose was based on higher cell 361 viability (Figure 1A). Pretreatment of cells with FLD $(50,75$ and $100 \mu \mathrm{g} / \mathrm{mL})$ for $18 \mathrm{~h}$ and 362 subsequently with $0.1 \mathrm{mM} t$-BHP for $3 \mathrm{~h}$ further enhanced catalase protein expression in a dose363 dependent manner as compared with $t$-BHP exposed cells ( $<<0.05$, Figure 2 B). Conversely, FLD 364 did not increased SOD-1 expression induced by $0.1 \mathrm{mM}$ pro-oxidant exposure $(\mathrm{p}>0.05$, Figure 365 2C).

\subsection{FLD induces the nuclear translocation of $N$ fr 2}

367 We investigated the effect of FLD in the expression and nuclear translocation of the transcription 368 factor Nrf2 by Western blot (Figure 2E). Accordingly, RAW 264.7 cells were pretreated with 369 FLD $(50,75$ and $100 \mu \mathrm{g} / \mathrm{mL})$ for $18 \mathrm{~h}$ and subsequently with $0.1 \mathrm{mM} t$-BHP for $3 \mathrm{~h}$ and $\mathrm{Nrf} 2$ in 370 nuclear fraction were measured by Western blot. Figure 2F shows a dose dependent increase of 371 Nrf2 levels in nuclear fraction $(\mathrm{p}<0.05)$.

372 3.6. FLD increases the phosphorylation of SAPK/JNK in RAW 264.7 macrophages

373 Because redox signaling mechanisms involve the activation of the stress-sensitive Ser/Thr 374 systems (Evans, Goldfine, Maddux, \& Grodsky, 2002), we examined the upstream signaling 375 pathways involved in the activation of Nrf2 by determination of phosphorylation of SAPK/JNK. 
376 RAW 264.7 cells were treated with $\operatorname{FLD}(50,75$ and $100 \mu \mathrm{g} / \mathrm{mL})$ for $6 \mathrm{~h}$ and Western blots were

377 performed to detect T- and P-SAPK/JNK proteins (Figure 3A). FLD-treated cells showed a 378 significant and dose-dependent increase of phosphorylation of SAPK/JNK that was evident after

$3796 \mathrm{~h}$ (Figure 3B). Conversely, no significant differences were observed in total levels of 380 SAPK/JNK of FLD treated cells compared to control cells ( $p>0.05$, Figure 3C). The ratio of 381 phosphorylated versus total SAPK/JNK kinase increased with FLD treatment in a dose 382 dependent manner $(\mathrm{p}<0.05$, Figure 3D).

\section{3.7. Characterization of FLD}

Most abundant peptides in FLD are presented in Table 2. A total of 7 fragments with molecular masses within the range of 839 to 2768 Da were identified. These peptides derived

386 from the major lentil proteins: convicilin, allergen Len c 1.0101 and vicilin type C. Table 3 387 shows the phenolic profile of FLD acquired by HPLC-ESI-DAD-MS ${ }^{\mathrm{n}}$ analysis in the negative388 ion mode. A total of 9 unique mass signals were detected ranging from 258-741 Da. Of these, 8 389 compounds were tentatively identified based on spectral database matching as well as literature 390 data (Dueñas et al., 2016). An unidentified compound with a mass/charge (m/z) ratio of 659 and 391 mass fragments of 468 and 298 was the most abundant compound, reaching up to $846.5 \mu \mathrm{g} / \mathrm{g}$ in

392 FLD. Phenolic acid derivatives such as feruloylquinic acid, gallic acid derivatives I and II and 393 caffeic acid glucuronide were the greatest phenolic group identified in FLD, reaching up to 88.9, $39472.1,56.9$ and $16.5 \mu \mathrm{g} / \mathrm{g}$, respectively. The second most abundant phenolic compounds identified 395 in FLD were the flavonols kaempferol rutinoside-rhamnoside and quercetin-glucuronide, which 396 reached 51.0 and $32.3 \mu \mathrm{g} / \mathrm{g}$, respectively.

\section{4. Discussion}

One of the purposes of this study was to scale up the production of FL and to investigate 399 the impact of pilot scale production on peptide and phenolic contents as well as on bioactivity. 
400 Experimental results indicated that fermentation scale up from $1.5 \mathrm{~L}$ to $30 \mathrm{~L}$ moderately reduced 401 the peptide and phenolic content as well as bioactivity of FL. These losses could be partially 402 associated to the fact that FL production at pilot scale was performed in milder alkaline 403 conditions ( $\mathrm{pH} 8.0)$ as compared to lab-scale production $(\mathrm{pH} 8.5)$. These small changes in $\mathrm{pH}$ 404 value could have a negative impact on Savinase protease activity (optimum $\mathrm{pH} 8.5$ ) and 405 subsequently on the generation of bioactive peptides, resulting in moderate losses of biological 406 effectiveness. The lower phenolic content found in the soluble fraction of fermented lentil 407 produced at pilot scale could be related to differences in fermentation conditions which may have 408 an impact in solubilization of bound phenolic fraction mediated by microbial metabolism, 409 Savinase (with feruloyl esterase side activity) and alkaline hydrolysis (Bautista-Exposito et al., 410 2018). Regarding antioxidant activity, what is suggested from our results is that upscale 411 production of fermented lentil slightly reduced the ability to scavenge peroxyl radicals through 412 hydrogen donation while improved inhibition of intracellular ROS production most likely 413 through activation of cellular antioxidant response. Antioxidant activity assays such as ORAC 414 and inhibition of $t$-BHP induced cellular oxidative stress provide different information about 415 mechanisms of action of antioxidant compounds. The former only measures the ability of an 416 antioxidant to quench free radicals (i.e. peroxyl radical) by hydrogen donation stabilizing the 417 peroxyl radical by resonance (Granato et al., 2018). The latter evaluates the ability of compounds 418 as modulators of cell signaling pathways related to activation of cellular antioxidant response 419 (Granato et al., 2018). In order to test this hypothesis the effect of fermented lentil after 420 gastrointestinal digestion on the activation of cellular antioxidant response was further examined. Despite the moderate losses in bioactivity observed in the present work, a recent study 422 demonstrated that FL produced in the same conditions described herein was efficient at 423 ameliorating alterations in glucose and lipid metabolism and liver functionality in an 424 experimental animal model of MetS and NAFLD (Martinez et al., 2018). In particular, FL was 
425 effective at improving all the parameters and indices related to insulin resistance (blood glucose,

426 area under the curve after an oral glucose overload and profile curve, triglyceride/glucose index),

427 lipid metabolism (plasmatic triglycerides, phospholipids and high density lipoprotein cholesterol

428 levels, atherogenic index), hepatic steatosis (fat content, fatty acid composition) and oxidative

429 stress (hepatic lipid peroxidation) in obese Zucker rats. These health benefits of FL were

430 consistent with the in vitro antioxidant, intestinal $\alpha$-glucosidase and pancreatic lipase inhibitory

431 activities observed in the present study for FL and it could be attributed to the presence of

432 bioactive peptides and phenolic compounds.

Accumulating scientific evidence demonstrates that MetS is associated with an impaired

434 antioxidant status due to a general decrease of hepatic antioxidant enzymes and subsequent

435 increase of lipid peroxidation in the liver (Martinez et al., 2018). Lipid peroxidation is the ROS-

436 triggered metabolic process that results in the destruction of membrane lipids and tissue damage.

437 Martinez et al. (Martinez et al., 2018) found that daily FL administration ( $1 \mathrm{~g} / \mathrm{kg}$ ) for 8 weeks

438 reverted hepatic lipid peroxidation in the obese Zucker rat. With that knowledge, a second

439 purpose of the current study was to investigate the protective effect of FL on $t$-BHP-induced

440 oxidative stress in RAW 264.7 macrophages. Moreover, we determined whether these effects

441 were associated to the activation of Nrf2-mediated antioxidant signaling pathways in RAW

442264.7 macrophages. In this study, we demonstrated that FLD was able to counteract $t$-BHP-

443 induced oxidative stress and cell death as well as to improve cellular antioxidant capacity of

444 macrophages by increasing the activity of catalase via a mechanism that involves activation of

$445 \mathrm{SAPK} / \mathrm{JNK}$ and Nrf2 nuclear translocation. Thus, our results show for the first time the

446 mechanisms of action of FLD to prevent oxidative damage in macrophages through the

447 modulation of signaling pathways involved in antioxidant enzyme regulation.

We selected RAW 264.7 macrophages because it is well known that this cell line could

449 own over-production of ROS following oxidant exposure (Zhang et al., 2018). Previous studies 
450 have shown that $t$-BHP exposure to RAW 264.7 macrophages reduced cell viability and

451 promoted cell apoptosis and necrosis resulting from intracellular ROS generation (Lv, Liu, Zhou,

452 Tan, Deng, \& Ci, 2017). Similarly, we found that RAW 264.7 cells exposed to $t$-BHP altered

453 intracellular redox milieu and decreased cell viability even at a relatively low level of $t$-BHP $(0.1$

$454 \mathrm{mM})$. We have shown herein that cell pretreatment with FLD prevented the increase in

455 intracellular ROS and promoted cell viability and survival of RAW 264.7 macrophages in a

456 strong oxidant environment $(2.5 \mathrm{mM} t$-BHP). These results are in agreement with an earlier study

457 showing that FL administration to obese Zucker rats decreases hepatic thiobarbituric reactive

458 species, a marker of lipid peroxidation (Martinez et al., 2018).

Accumulating evidence shows that cytoprotective effect of antioxidants may be linked to

460 their ability to regulate the expression of antioxidant enzymes (Ramachandran \& Saravanan,

461 2013). Expression of antioxidant enzymes is critical in the detoxication and elimination of ROS

462 and electrophiles through conjugative reactions and by enhancing cellular antioxidant capacity.

463 Our results revealed that pretreatment of RAW 264.7 macrophages with FLD and subsequent

464 exposure to oxidant conditions enhanced the expression of catalase in RAW 264.7 macrophages

465 as compared to non-stressed cells. Catalase catalyzes the conversion of hydrogen peroxide to

466 water and oxygen (Finkel \& Holbrook, 2000). Therefore, we proposed that the increased

467 endogenous defense capacity evoked by FLD could be a critical determinant to protect against

468 oxidative stress-induced injury. Similarly, early studies have found that legume (Phaseolus

469 vulgaris L.) extracts displayed protective benefit to endogenous antioxidant enzymes such as

470 SOD, catalase, glutathione peroxidase and glutathione reductase in human colonic carcinoma

471 Caco-2 cells (Chen, Zhang, Marcone, Pauls, Liu, Tang, et al., 2017).

472 A major mechanism in the cellular defense against oxidative stress is activation of the

473 Nrf2-antioxidant response element (ARE) signaling pathways (Nguyen, Nioi, \& Pickett, 2009).

474 Activation of $\mathrm{Nfr} 2$ is dependent on mechanisms that increase its stability, leading to its 
475 accumulation in the cell. A well stablished mechanism that controls Nrf2 stability is mediated

476 by Keap1 (Nguyen, Sherratt, Nioi, Yang, \& Pickett, 2005). Nrf2 is targeted for degradation by

477 Keap1 in the nucleus, a process that requires the transient suttling of Keap1 into this

478 compartment. In cells under stress, stabilization of Nrf2 is thought to be dependent on

479 modifications of reactive cysteines in Keap 1 that reduce access of this protein to $\mathrm{Nrf} 2$

480 (Kobayashi et al., 2006). We investigated the mode of action of FLD to induce the antioxidant

481 defense via Nrf2 activation in RAW 264.7 macrophages. In the present study, we observed that

482 FLD pretreatment of cells clearly induces the nuclear translocation and dose-dependent

483 accumulation of Nrf2, a necessary event to exert its activity. These results were consistent with

484 the increase of catalase gene expression and suggest a clear regulatory mechanism promoting

485 Nrf2 stabilization in response to FLD and induction of ARE-mediated antioxidant enzymes gene

486 expression. Similarly, fermented powder of common bean (Phaseolus vulgaris L.) was able to

487 regulate antioxidant and detoxifying enzymes through the $\mathrm{Nrf} 2$ pathway, and reduce $\mathrm{H}_{2} \mathrm{O}_{2}-$

488 induced endoplasmic reticulum (ER) stress (La Marca et al., 2015).

Nrf2 activation by phosphorylation at specific serine and/or tyrosine residues has also

490 been reported to be mediated by upstream signaling pathways such as mitogen activated protein

491 kinases (MAPK), phosphatidylinositol-3-kinases/protein kinase B or protein kinase C $\delta$ (Lv, Liu,

492 Zhou, Tan, Deng, \& Ci, 2017; Niture, Khatri, \& Jaiswal, 2014). As with other members of the

493 MAPK family, JNK is activated upon dual phosphorylation on a Thr-Pro-Tyr motif, which is

494 activated by phosphorylation on serine and threonine residues by an upstream kinase (MKK4

495 and MKK7) (Davis, 1999). Therefore, to identify upstream regulatory mechanisms involved in

496 the nuclear accumulation of Nfr2 by FLD, the effect on the phosphorylated levels of SAPK/JNK

497 were also examined in the present study. We found that FLD induced the phosphorylation of

498 SAPK/JNK, therefore, these results suggest that the activation of this pathway could be linked

499 to Nfr2 activation. The present study demonstrates for the first time that FLD induces the 
expression of antioxidant enzymes through a mechanism involving activation of Nfr2/MAPK 501 pathways.

Lentil is a source of bioactive peptides and phenolic compounds with antioxidant activity,

503 therefore, the last purpose of the present study was to stablish a relationship between the 504 cytoprotective effect of FLD against $t$-BHP induced oxidative stress in RAW 264.7 macrophages 505 and FLD peptide and phenolic composition. HPLC-MS/MS analysis allowed the identification 506 of 7 peptides in FLD derived from the main lentil proteins (convicilin, convicilin, allergen Len 507 c 1.0101 and vicilin type C). Two of these sequences derived from convicilin 508 (EQSPGQWRPSHGKEEDEEEK and EQSPGQWRPSHGKEEDEEEKEQK) were previously

509 found in FL which was indicative of the resistance of these peptides to in vitro gastrointestinal 510 digestion simulating physiological conditions (Bautista-Exposito et al., 2018b). Structure511 activity relationship analysis revealed that amino acid sequences containing proline, histidine or 512 tyrosine within the sequence and hydrophobic amino acids at the N-terminus and/or C-terminus 513 are determinant for the antioxidant activity ( $\mathrm{Li} \& \mathrm{Li}, 2013)$. Moreover, it has been shown that 514 amino and carboxylic group of acidic (aspartic acid and glutamic acid) and basic (arginine, lysine 515 and histidine) amino acids act as metal chelators (Díaz, Dunn, McClements, \& Decker, 2003).

516 These structural features were found in most of the peptides collected in Table 2. Therefore, it 517 can be assumed that lentil derived peptides in FLD identified in the present study could have 518 contributed to reduction of intracellular ROS levels in $t$-BHP-induced macrophages through their 519 radical scavenging activity. In addition, there is accumulating evidence pointing out that legume 520 derived oligopeptides could exert an indirect antioxidant mechanism through the activation of 521 the cellular antioxidant system. In particular, the chickpea-derived peptide NRYHE upregulates 522 Nrf2 expression and participates in the ARE-mediated activation of antioxidant enzymes 523 including $\mathrm{NADP}(\mathrm{H})$ :quinone oxidoreductase 1 , heme oxygenase $1, \gamma$-glutamyl cysteine 524 synthetase in $\mathrm{H}_{2} \mathrm{O}_{2}$-treated HT-29 and Caco-2 cells (Guo, Zhang, Jiang, Miao, \& Mu, 2014). 
The antioxidant activity of lentil derived peptides can be potentiated by the variety of

526 phenolic compounds identified in FLD. Phenolic acids were the most prominent phenolic 527 compounds in FLD followed by flavonols. Our results contrast with the phenolic profile of non-

528 digested FL that is mainly made up of flavonols (kaempferol aglycone and glucosides,

529 isorhamnetin glucuronide), followed by flavan-3-ols [(+)-catechin-3-O-glucoside,

530 prodelphinidin dimer and $(+)$-catechin] and minor amounts of hydroxycinnamic and

531 hydroxybenzoic compounds (Bautista-Exposito et al., 2018b). The differences observed in the

532 phenolic profile of FLD could be attributed to instability of flavonols and flavan-3-ols to $\mathrm{pH}$

533 changes during the different stages of gastrointestinal digestion (Bautista-Exposito et al., 2018b).

534 Earlier studies have shown that phenolic compounds present in FLD are able to neutralize

535 radicals and chelate metal ions through hydrogen, electron donating and mixed mechanisms

536 (Ganesan \& Xu, 2017). However, only some of them have been proven as Keap1-Nrf2 activators.

537 For instance, kaempferol aglycone and glucosides have shown to exert protective effects against

538 oxidative stress through activation of Nrf2-mediated defensive response in lung tissues (Kim,

539 Im, Lee, \& Chae, 2017; Kumar, Kim, More, Kim, \& Choi, 2014). Similarly, gallic acid, quercetin

540 and quercetin glucoside protects against oxidative stress-induced hepatotoxicity by activation of

541 the Keap1-Nrf2 pathway (Feng, Wang, He, Yang, \& Wan, 2017; Joko, Watanabe, Fuda, Takeda,

542 Furukawa, Hui, et al., 2017; Kim, Im, Lee, \& Chae, 2017). Structure-activity relationship

543 analysis revealed that compounds bearing phenolic hydroxyl groups are bifunctional

544 antioxidants that exert protective role by scavenging hazardous oxidants and inducing

545 cytoprotective enzymes (Dinkova-Kostova \& Talalay, 2008).

\section{5. Conclusions}

547 In summary, we have shown that FLD composed of antioxidant peptides and phenolic 548 compounds is able to enhance the cellular antioxidant defense capacity, thereby, protecting 549 macrophages from oxidative stress. FLD reverted t-BHP-induced cytotoxicity and ROS 
550 production through activation of SAPK/JNK phosphorylation followed by nuclear accumulation

551 and transactivation and the subsequent expression of catalase. All these events were essential to 552 maintain cellular redox homeostasis and prevent oxidative stress cell damage.

\section{Funding sources}

554 The research leading to these results received funding from Ministry of Economy and 555 Competitiveness (MINECO, Spain) and FEDER program [grant number AGL2013-43247-R]

556 and Spanish National Research Council [grant number 201670I044]. E. P. acknowledges to

557 MINECO and European Social Fund for her "Ramón y Cajal" contract.

558 Conflicts of interest: The authors declare no conflict of interest.

\section{ABBREVIATION LIST}

560 ACE: angiotensin I converting enzyme inhibitory activity; ACN: acetonitrile; ARE: antioxidant 561 response element; $t$-BHP: tert-butyl hydroperoxide; DCF: dichlorofluorescein; DCFDA: 562 dichlorofluorescein diacetate; DMEM: High-glucose Dulbecco's Modified Eagle's Medium; 563 FA: formic acid; FBS: Fetal bovine serum; FL: fermented lentil; FLD: fermented lentil digest; 564 HRP: horse radish peroxidase; JNK: Jun amino-terminal kinases; NAFLD: non-alcoholic fat

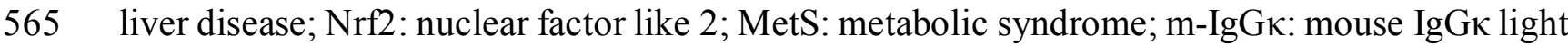
566 chain binding protein; ORAC: oxygen radical absorbance capacity; PBS: phosphate buffer 567 saline; PVDF: Polyvinylidene fluoride; ROS: reactive oxygen species; SAPK-stress-activated 568 protein kinases; SOD-1: $\mathrm{Cu}-\mathrm{Zn}$ superoxide dismutase; TFA: trifluoroacetic acid; TBST: Tris 569 buffer saline Tween 20 


\section{References}

Bautista-Exposito, S., Peñas, E., Silván, J. M., Frias, J., \& Martínez-Villaluenga, C. (2018a). pHcontrolled fermentation in mild alkaline conditions enhances bioactive compounds and functional features of lentil to ameliorate metabolic disturbances. Food Chemistry, 248, 262271.

Bautista-Exposito, S., Peñas, E., Dueñas, M., Silván, J.M., Frias, F., Martínez-Villaluenga, C. (2018b). Individual contributions of Savinase and Lactobacillus plantarum to lentil functionalization during alkaline pH-controlled fermentation. Food Chemistry, 257, 341-349.

Bazzano, L. A., Green, T., Harrison, T. N., \& Reynolds, K. (2013). Dietary approaches to prevent hypertension. Current Hypertension Reports, 15(6), 694-702.

Chen, P. X., Zhang, H., Marcone, M. F., Pauls, K. P., Liu, R., Tang, Y., Zhang, B., Renaud, J. B., \& Tsao, R. (2017). Anti-inflammatory effects of phenolic-rich cranberry bean (Phaseolus vulgaris L.) extracts and enhanced cellular antioxidant enzyme activities in Caco-2 cells. Journal of Functional Foods, 38, 675-685.

Clark, S., \& Duncan, A. M. (2017). The role of pulses in satiety, food intake and body weight management. Journal of Functional Foods, 38, 612-623.

Davis, R. J. (1999). Signal transduction by the c-Jun N-terminal kinase. In Biochemical Society Symposium, vol. 64 (pp. 1-12).

Díaz, M., Dunn, C. M., McClements, D. J., \& Decker, E. A. (2003). Use of caseinophosphopeptides as natural antioxidants in oil-in-water emulsions. Journal of Agricultural and Food Chemistry, 51(8), 2365-2370.

Dinkova-Kostova, A. T., \& Talalay, P. (2008). Direct and indirect antioxidant properties of inducers of cytoprotective proteins. Molecular Nutrition and Food Research, 52(SUPPL. 1), S128S138. 
594 Dongiovanni, P., Lanti, C., Riso, P., Valenti, L. (2016). Nutritional therapy for non-alcoholic fatty liver disease. Journal of Nutritional Biochemistry, 29, 1-11.

Dueñas, M., Sarmento, T., Aguilera, Y., Benitez, V., Mollá, E., Esteban, R. M., \& Martín-Cabrejas, M. A. (2016). Impact of cooking and germination on phenolic composition and dietary fibre

Evans, J. L., Goldfine, I. D., Maddux, B. A., \& Grodsky, G. M. (2002). Oxidative stress and stressactivated signaling pathways: A unifying hypothesis of type 2 diabetes. Endocrine Reviews, 23(5), 599-622.

Feng, R. B., Wang, Y., He, C., Yang, Y., \& Wan, J. B. (2017). Gallic acid, a natural polyphenol, protects against tert-butyl hydroperoxide- induced hepatotoxicity by activating ERK-Nrf2Keap1-mediated antioxidative response. Food and Chemical Toxicology.

Finkel, T., \& Holbrook, N. J. (2000). Oxidants, oxidative stress and the biology of ageing. Nature, 408(6809), 239-247.

Ganesan, K., \& Xu, B. (2017). Polyphenol-rich lentils and their health promoting effects. International Journal of Molecular Sciences, 18(11).

Garcia-Mora, P., Frias, J., Peñas, E., Zieliński, H., Giménez-Bastida, J. A., Wiczkowski, W., Zielińska, D., \& Martínez-Villaluenga, C. (2015). Simultaneous release of peptides and phenolics with antioxidant, ACE-inhibitory and anti-inflammatory activities from pinto bean (Phaseolus vulgaris L. var. pinto) proteins by subtilisins. Journal of Functional Foods, 18, Part A, 319-332.

García-Mora, P., Martín-Martínez, M., Angeles Bonache, M., González-Múniz, R., Peñas, E., Frias, J., \& Martinez-Villaluenga, C. (2017). Identification, functional gastrointestinal stability and molecular docking studies of lentil peptides with dual antioxidant and angiotensin I converting enzyme inhibitory activities. Food Chemistry, 221, 464-472. 
Granato, D., Shahidi, F., Wrolstad, R., Kilmartin, P., Melton, L.D., Hidalgo, F.J., Miyashita, K., Camp, J.V., Alasalvar, C., Ismail, A.B., Elmore, S., Birch, G.G., Charalampopoulos, D., Astley, S.B., Pegg, R., Zhou, P., Finglas, P. (2018). Antioxidant activity, total phenolics and flavonoids contents: Should we ban in vitro screening methods? Food Chemistry, 264, 471475.

Grundy, S. M. (2016). Metabolic syndrome update. Trends in Cardiovascular Medicine, 26(4), 364373.

Guo, Y., Zhang, T., Jiang, B., Miao, M., \& Mu, W. (2014). The effects of an antioxidative pentapeptide derived from chickpea protein hydrolysates on oxidative stress in Caco-2 and HT-29 cell lines. Journal of Functional Foods, 7(1), 719-726.

Iwaniak, A., Darewicz, M., \& Minkiewicz, P. (2018). Peptides Derived from Foods as Supportive Diet Components in the Prevention of Metabolic Syndrome. Comprehensive Reviews in Food Science and Food Safety, 17(1), 63-81.

Joko, S., Watanabe, M., Fuda, H., Takeda, S., Furukawa, T., Hui, S.-P., Shrestha, R., \& Chiba, H. (2017). Comparison of chemical structures and cytoprotection abilities between direct and indirect antioxidants. Journal of Functional Foods, 35, 245-255.

Kapravelou, G., Martínez, R., Nebot, E., López-Jurado, M., Aranda, P., Arrebola, F., Cantarero, S., Galisteo, M., \& Porres, J. M. (2017). The combined intervention with germinated vigna radiata and aerobic interval training protocol is an effective strategy for the treatment of nonalcoholic fatty liver disease (NAFLD) and other alterations related to the metabolic syndrome in zucker rats. Nutrients, $9(7)$.

Kim, K. M., Im, A. R., Lee, S., \& Chae, S. (2017). Dual protective effects of flavonoids from Petasites japonicus against UVB-induced apoptosis mediated via HSF-1 activated heat shock proteins and Nrf2-activated heme oxygenase-1 pathways. Biological and Pharmaceutical Bulletin, $40(6), 765-773$. 
Kobayashi, A., Kang, M. I., Watai, Y., Tong, K. I., Shibata, T., Uchida, K., \& Yamamoto, M. (2006). Oxidative and electrophilic stresses activate Nrf2 through inhibition of ubiquitination activity of Keap1. Molecular and Cellular Biology, 26(1), 221-229.

Kumar, H., Kim, I. S., More, S. V., Kim, B. W., \& Choi, D. K. (2014). Natural product-derived pharmacological modulators of Nrf2/ARE pathway for chronic diseases. Natural Product Reports, 31(1), 109-139.

La Marca, M., Pucci, L., Bollini, R., Russo, R., Sparvoli, F., Gabriele, M., \& Longo, V. (2015). Antioxidant effect of a fermented powder of Lady Joy bean in primary rat hepatocytes. Cellular and Molecular Biology Letters, 20(1), 102-116.

Li, Y. W., \& Li, B. (2013). Characterization of structure-antioxidant activity relationship of peptides in free radical systems using QSAR models: Key sequence positions and their amino acid properties. Journal of Theoretical Biology, 318, 29-43.

Lv, H., Liu, Q., Zhou, J., Tan, G., Deng, X., \& Ci, X. (2017). Daphnetin-mediated Nrf2 antioxidant signaling pathways ameliorate tert-butyl hydroperoxide (t-BHP)-induced mitochondrial dysfunction and cell death. Free Radical Biology and Medicine, 106, 38-52.

Martinez, R., Kapravelou, G., Donaire, A., Lopez-Chaves, C., Arrebola, F., Galisteo, M., Cantarero, S., Aranda, P., Porres, J. M., \& Lopez-Jurado, M. (2018). Effects of a combined intervention with a lentil protein hydrolysate and a mixed training protocol on the lipid metabolism and hepatic markers of NAFLD in Zucker rats. Food \& Function, 9, 830-850.

Martínez, R., López-Jurado, M., Wanden-Berghe, C., Sanz-Valero, J., Porres, J. M., \& Kapravelou, G. (2016). Beneficial effects of legumes on parameters of the metabolic syndrome: A systematic review of trials in animal models. British Journal of Nutrition, 116(3), 402-424.

Minekus, M., Alminger, M., Alvito, P., Ballance, S., Bohn, T., Bourlieu, C., Carrière, F., Boutrou, R., Corredig, M., Dupont, D., Dufour, C., Egger, L., Golding, M., Karakaya, S., Kirkhus, B., Le Feunteun, S., Lesmes, U., MacIerzanka, A., MacKie, A., Marze, S., McClements, D. J., Ménard, O., Recio, I., Santos, C. N., Singh, R. P., Vegarud, G. E., Wickham, M. S. J., 
Weitschies, W., \& Brodkorb, A. (2014). A standardised static in vitro digestion method suitable for food-an international consensus. Food and Function, 5(6), 1113-1124.

Mollard, R. C., Luhovyy, B. L., Panahi, S., Nunez, M., Hanley, A., \& Anderson, G. H. (2012). Regular consumption of pulses for 8 weeks reduces metabolic syndrome risk factors in

Padhi, E. M. T., \& Ramdath, D. D. (2017). A review of the relationship between pulse consumption and reduction of cardiovascular disease risk factors. Journal of Functional Foods, 38, 635643.

Ramachandran, V., \& Saravanan, R. (2013). Asiatic acid prevents lipid peroxidation and improves antioxidant status in rats with streptozotocin-induced diabetes. Journal of Functional Foods, 5(3), 1077-1087. 
694 Reverri, E. J., Randolph, J. M., Kappagoda, C. T., Park, E., Edirisinghe, I., \& Burton-Freeman, B. M. 695 (2017). Assessing beans as a source of intrinsic fiber on satiety in men and women with 696 metabolic syndrome. Appetite, 118, 75-81.

697 Zaki, M., Kamal, S., Basha, W. A., El-Toukhy, S., Yousef, W., El-Bassyouni, H. T., \& Azmy, O. 698 (2018). Assessment of DNA damage in obese premenopausal women with metabolic 699 syndrome. Gene Reports, 10, 42-46.

700 Zhang, B., Deng, Z., Ramdath, D. D., Tang, Y., Chen, P. X., Liu, R., Liu, Q., \& Tsao, R. (2015). 701 Phenolic profiles of 20 Canadian lentil cultivars and their contribution to antioxidant activity 702 and inhibitory effects on $\alpha$-glucosidase and pancreatic lipase. Food Chemistry, 172, 862-872. 703 Zhang, X.-T., Sun, X.-Q., Wu, C., Chen, J.-L., Yuan, J.-J., Pang, Q.-F., \& Wang, Z.-P. (2018). Heme 704 oxygenase-1 induction by methylene blue protects RAW264.7 cells from hydrogen peroxideinduced injury. Biochemical Pharmacology, 148, 265-277. 
Figure 1. FLD reduces $t$-BHP-induced cell death (A-C) and intracelullar redox status (D-F) in

RAW 264.7 macrophages. RAW 264.7 macrophages were exposed to $t$ - $\mathrm{BHP}(0.05,0.1,1.0$ and 2.5 $\mathrm{mM})$ for $3 \mathrm{~h}(\mathrm{~A}$ and D) FLD (50, 75 and $100 \mu \mathrm{g} / \mathrm{mL})$ for $18 \mathrm{~h}(\mathrm{~B}$ and E) and FLD (50, 75 and 100 $\mu \mathrm{g} / \mathrm{mL}$ ) for $18 \mathrm{~h}$ followed by treatment with $2.5 \mathrm{mM} t$-BHP for $3 \mathrm{~h}(\mathrm{C}$ and $\mathrm{F})$. Cell viability was measured using a tetrazolium compound [3-(4,5-dimethylthiazol-2-yl)-5-(3carboxymethoxyphenyl)-2-(4-sulfophenyl)-2H-tetrazolium, inner salt] and an electron coupling reagent (phenazine ethosulfate). Cell viability was expressed as relative percentage of control cells.

714 Oxidant production was measured by DCF probe. Data were expressed relative percentage of 715 fluorescence intensity in control cells. All results are expressed as means \pm standard deviation of six independent experiments for each experimental condition. Different letters denote statistically significant differences $(\mathrm{p}<0.05$, Duncan test).

Figure 2. FLD activates catalase protein expression (A-C) and Nrf2 nuclear translocation (D and E) in RAW 264.7 macrophages. RAW 264.7 macrophages were treated with FLD (50, 75 and $100 \mu \mathrm{g} / \mathrm{mL}$ ) for $18 \mathrm{~h}$ followed by $0.1 \mathrm{mM} t$-BHP for $3 \mathrm{~h}$. Following treatment, protein was harvested and immunoblotted for the indicated proteins. Representative Western blot of three different experiments (A and D). Protein expression of catalase, SOD-1 and Nrf2 were relative to untreated cells (B, C and F respectively). The intensities of catalase, SOD-1, Nfr2, Lamin B1 and $\beta$-actin was quantified using Bio-Rad Image Lab software. All results were expressed as means \pm standard deviation of three independent experiments. Different letters denote statistically significant differences ( $p<0.05$, Duncan test).

Figure 3. FLD protective effect against oxidative stress involves MAPK activation. Total (T) and phosphorylated (P) SAPK/JNK were detected by Western blot in cytosolic lysates after RAW 264.7 macrophages treatment with FLD $(50,75$ and $100 \mu \mathrm{g} / \mathrm{mL})$ for $6 \mathrm{~h}$. Following treatment, protein was harvested and immunoblotted for the indicated proteins. Representative Western blot image of T- 
$731 \mathrm{SAPK} / \mathrm{JNK}$ and P-SAPK/JNK (A). T-SAPK/JNK and P-SAPK/JNK intensity relative to $\beta$-actin 732 expressed as percentage relative to control cells (B). Ratio of P-SAPK/JNK vs. T-SAPK/JNK (C). 733 The intensities of T-SAPK/JNK and P-SAPK/JNK and $\beta$-actin were quantified using Bio-Rad Image 734 Lab software. All results were expressed as means \pm standard deviation of three independent 735 experiments. Different letters denote statistically significant differences $(p<0.05$, Duncan test). 736 737 738 